\title{
Low Cost Heritage Imaging Techniques Compared
}

\author{
Moshe Caine \\ Hadassah Academic College Jerusalem \\ 37 Hanevi'im Street, Jerusalem, Israel \\ mosheca@hadassah.ac.il
}

\author{
Michael Magen \\ Israel Museum Jerusalem \\ Derech Ruppin, Jerusalem, Israel \\ michaelm@imj.org.il
}

\begin{abstract}
This paper challenges the notion that cultural heritage imaging technologies can only be the domain of advanced heritage institutions and must necessarily involve expensive equipment. Three main technologies are examined and compared: Reflectance Transformation Imaging, photogrammetry and low-end 3D scanning techniques. These are compared in regard of their ease of use in real-life situations, accuracy, reliability and flexibility in covering a multitude of heritage documentation cases. A variety of cases studies are presented where tangible heritage objects were imaged using two or more techniques and results were compared. The paper proposes that there is no clear winner and that there is not a single solution for all cases. Each technology has its place, its relative advantages and disadvantages. It suggests that heritage institutions can and should embrace all three, while empirically gauging their effectiveness in their local environment and specific needs. Finally, it is suggested that considering the rapid and continual advancement in technology, quality will continue to rise and prices may well drop. However, training and expertise will not replace the promise of automated solutions.
\end{abstract}

Culture. Heritage. Imaging. RTI. 3D. Photogrammetry. Scanning.

\section{INTRODUCTION}

Conservation of tangible cultural heritage $(\mathrm{CH})$ properties involves a vast range of considerations. This paper focuses on one such area, that of visual inspection for curatorial documentation, quality maintenance, degradation charting and as an aid in conservation assessment and art in transit.

Over the past decade massive improvements have been made in visual technologies for imaging in both the human and the multi-spectral range. High definition laser and structured light scanners have brought scientific exactitude to the realm of conservation as too have advances in MRI, thermal and other methods of imaging. Likewise, much research has focused upon comparative analysis of the merits of laser and structured light scanning as well as on photogrammetry. (Boehler et al. 2004; Kadobayashi et al. 2004; Grussenmeyer et al. 2008). Unfortunately, most of these technologies lie outside the reach of most museums and conservation institutes, due to cost of the hardware as well as to the level of expertise required for the running of such equipment.

Today's market demands low cost and practical solutions to combat the dangers of losing out to the ravages of decay, war, vandalism destruction, terrorism and time. (Reznicek, Pavelka 2007).

Likewise, some argue the value of simple methodologies and advocate bridging the gap between technologists and content specialists (Hassani 2015) (Scopigno et al. 2011).

In this paper, we have chosen to perform a comparative analysis of three low cost technologies: RTI (Reflectance Transformation Imaging), Photogrammetry - SFM, and low cost 3D scanning.

Not all heritage objects are equal in terms of size, materials, intricacy, colour-fidelity, texture, ease of access, etc. Therefore, it is not reasonable to expect one imaging solution to answer all visual documentation needs. Nor is it our aim to recommend one technique as an all-round winner or as a "one size fits all" solution. Rather, this paper attempts to outline the relative strengths and weaknesses, advantages and disadvantages of the different methods in a variety of circumstances and to suggest practical and empirical solutions for the clear majority of institutions who lack the luxury of high end imaging services. 


\section{2D-3D RATIONALE}

It may be argued that the inclusion of RTI technology within this comparative report is not valid or indeed correct.

RTI is clearly not a real 3D technology, it shares none of the characteristics of triangulation based photogrammetry, nor those of active scanning, by laser, structured light or Time of Flight scanners. Without delving deeply into the science, it should be noted that the two differ in their mathematical approach. RTI is a 2D detailed mathematical map of the surface texture, based on the normal vectors, while photogrammetry is three-dimensional point cloud and mesh based on trigonometric triangulation.

However, it can be said that the inclusion of this excellent technology is indeed relevant since our purpose is an empirical evaluation of existing low cost approaches to achieving visual surface or volume information from $\mathrm{CH}$ objects. The way we achieve this data is not necessarily the issue, rather the validity and applicability of the information. For many purposes, it will be argued, the practicality, accuracy and immediacy of RTI places it alongside $3 \mathrm{~d}$ imaging techniques as an equal among equals, and in some cases, more than that.

Furthermore, if we compare the techniques used in obtaining the final representation, we will see that in some respects, RTI is more like Photogrammetry that active scanning is. For one thing, both are computational photographic image based techniques. Both are based on passive image acquisition, whereas scanning is non-photographic and uses active light projection, be it blue/white, IR or laser light.

In addition, the actual acquisition methods applied in RTI and photogrammetry are remarkably similar. They both rely on multiple images, where, In the one case (RTI), the object and camera are static and the light source rotates. In the other case (Photogrammetry/SFM) the light and object are static while it is the camera which rotates.

\section{TECHNOLOGIES}

The following technologies were used for this empiric research:

\subsection{Reflectance Transformation Imaging}

- Highlight based technique - HSH Fitter

- Dome LP - HSH Fitter

\subsection{Photogrammetry}

- Target calibration mat technique - 3D SOM

- Silhouette Mask - Stereo Point-cloud Generation - 3D SOM

- Structure from Motion (SfM) - Agisoft PhotoScan

- SfM - Autodesk Recap 360

- SfM - Autodesk Remake

\subsection{D Scanning}

While good 3D scanners are still way beyond the budget of the average small museum and conservation institute, they are proliferating in shape and size and the prices are falling. Nevertheless, in keeping with the proposed intention of "low cost" solutions, three scanner solutions were used.

- The NextEngine Laser scanner - At $\$ 3000$, this lies at the top edge of the "low price" scale.

- The Cubify Sense handheld IR depth scanner (the scanner projects a patterned infrared beam onto the object that is then detected by a built-in webcam.)

- Microsoft Kinect IR depth scanner + open source software

\section{TECHNOLOGY OVERVIEW}

Where possible, each object was imaged using three alternative techniques: RTI, Photogrammetry and 3D Scan. Each of the three techniques was likewise performed with various hardware and software in accordance with the limitations of size, environment, access and complexity.

Following is a summary of the principle technologies. Obviously, each one could and should be explained in much greater detail. This overview is provided primarily for those less familiar with the topics and as a background for the decision-making process in the empirical tests carried out.

\subsection{RTI - Reflectance Transformation Imaging}

Originally termed Polynomial Texture Mapping (PTM), Reflectance Transformation Imaging was invented by Tom Malzbender at the Hewlett Packard Labs. The seminal paper describing the method was published in 2001(Malzbender, Gelb, Wolters 2001). Today the technology continues to be developed by a dedicated international team, supported by the Cultural Heritage Imaging Corporation (CHI).

RTI is a unique digital photographic technique aimed at enhancing the surface detail of objects through an algorithmic rendering of multiple 
registered digital images of the object, shot with controlled, varying, yet known light positions.

The light sources are positioned at a constant radius from the subject and surround it at incremental angles, forming a dome or hemisphere of light positions.

The photographs, anywhere between $40-70$, are then fed into software which calculates the light positions (L.P. file) and forms the basis for the creation of the final PTM file, essentially a virtual three-dimensional digital image light source that is controlled interactively. The final image, viewed with a Java based viewer (RTI viewer), initially looks like a flat, normal photograph. The magic however starts with the ability of the user to intuitively and interactively move the virtual light source around the image by moving the mouse in a manner that MacDonald terms a 'virtual torch' (MacDonald 2011), zoom in and out, and change sharpness, contrast and other light and surface properties through a series of real time filters, thus often revealing surface details not visible to the naked eye under normal viewing conditions.

\subsection{Photogrammetry - Photo Modelling}

Photogrammetry is the "science of measuring in photos", and is most commonly used in remote sensing, aerial photography, archaeology, architecture and other fields where we need to determine measurements from photographs.

It is based on the principle that while a single photograph can only yield two-dimensional coordinates, height and width (X-Y), two overlapping images of the same scene, taken slightly apart from each other can allow the third dimension $(Z)$ to be calculated through the process of triangulation.

Photo modelling is the process of creating a true 3D model via a combined methodology of photographic and true vector modelling techniques.

A photo model represents the shape, texture and lighting of the object as a list of points in Cartesian space. Each point is represented on the three axes, $X, Y$ and $Z$. The more points representing the object, the greater the accuracy and detail achievable.

In Photo Modelling the object is photographed from all angles to obtain ample and overlapping visual coverage of the entire surface.

The beauty and relevance of the photographic approach is that while the conventional 3D models are created from scratch, these models are created by photography. In other words, rather than "making" a picture we are "taking" a picture. This process lends itself especially to $3 D$ reproduction and representation of existing real things, rather than the creation process of new objects, based on copying or imagination. Furthermore, certain objects may prove immensely difficult to recreate in conventional 3D, especially objects with soft, amorphous, non-geometric shapes. This is where photo modeling stands out.

The past several years have seen a great advancement in this field, due both to improvements in algorithms and software, as well in the proliferation of high quality DSLR cameras and even high end phone cameras. Consequently, there is today a growing argument that for many purposes, image based photogrammetry can equal and often surpass laser and SL imaging (Skarlatos \& Kiparissi 2012). Moreover, Pavelka argues that with good configuration and quality photos taken by digital camera, the accuracy (standard deviation) reaches from 0.2 to $1 \mathrm{~mm}$ from distances $1-5$ meters. In larger and more distant objects the accuracy is worse, but still acceptable. (Pavelka et al. 2013)

\subsection{Low cost 3D Scanning}

Conventional 2D Flatbed scanners are basically a digital camera without a camera optical system. The image data is gathered by light beamed on and reflected off a (usually) two-dimensional subject. The gathered light intensity is translated into pixel density. Thus, very high resolution bitmap images can be created, albeit of static subjects which can fit into the scan area.

However, because 2D scanners have no optical system to speak of, they can focus only on a set distance and measure only the $X$ and $Y$ coordinates of the points. Thus, they are not suitable for three dimensional objects of any considerable depth. This is where $3 \mathrm{D}$ scanners differ. They also gather the Z coordinate. All 3D scanners gather information of the object or environment scanned. This includes shape, volume and sometimes also texture and colour. They do this by a variety of methods which distinguish the various types of scanners.

\subsubsection{Laser Scanners}

The term Laser scanning has become synonymous with $3 \mathrm{~d}$ scanning. However, the laser is but one of the technologies in use today. Simply put, laser scanners project a point or beam of light onto the subject surface and measure the reflection of the light, thereby calculating the position and distance. Each point of light data is then aligned with the other multitude of points (possibly millions) to form a point cloud, similar, but usually of greater accuracy than that created by the photo modelling method.

Laser scanners are usually divided into two main groups: Contact Lasers and Non-Contact Laser. 
As the name implies, contact lasers come into direct contact with the surface area of the object scanned. For industrial design prototyping, reverse engineering and many other uses this allows extremely high levels of accuracy. However, this system is not always acceptable or even possible for some objects. For example, many cultural heritage objects are far too fragile to allow for this kind of treatment.

"Time of Flight" scanners measure the time it takes for the light to reflect from the subject. This is useful for measuring large objects at a distance, such as architectural structures, archaeological sites and the such. Their accuracy however is limited, usually to within a few millimeters.

Some laser scanners calculate point by point. Others shine a narrow beam or strip of light to speed up the data gathering process.

Like all technologies, Laser scanners have their advantages and disadvantages.

Wachowiak \& Karas (2013) argue the advantages of using advanced 3D laser and structured light scanners such as the Breuckmann triTOS-HE due to their high accuracy, yet even these scanners fall short when good quality colour texture is required. In addition, their high cost place them well outside the scope of the conditions set in this article (Wachowiak \& Karas 2013).

In this research, we have used the NextEngine scanner which is recognized as being a leader in the low end low cost range. This scanner includes a 3D colour capture capability, but as we shall see, it is if very low quality and not of much practical use for the fidelity required for heritage documentation.

Because 3D scanners map points in space only, they do not create highlights or shadows. Whilst this may seem a limitation for 3D viewing, it is in fact an advantage, as many if not most $3 \mathrm{D}$ viewing platforms offer a controllable lighting, thus allowing a fully interactive relighting of the object as needed. Research into the comparative qualities of SfM and $3 \mathrm{D}$ scanning in various applications is growing, such as in archaeology (P. Bourke) and cultural monuments (B. Bayram et al.).

\subsubsection{Structured Light Scanners}

Structured-light 3D scanners are similar in principle to triangulation scanners. However, they do not use a laser, but rather white (or blue) light.

Structured light scanners project a line or a pattern of light on the subject and look at the deformation of the pattern on the subject. The line is projected onto the subject using either an LCD projector or a sweeping laser. A camera is used to look at the deformation of the pattern, and an algorithm is used to calculate the distance at each point in the pattern. Unlike the time of flight scanners, triangulation scanners usually work best at relatively short distances and on small to medium sized objects.

It is argued that the use of structured light is better suited to cultural heritage objects than laser light (Akca 2012) It reduces speckle noise and provides better surface smoothness (Blais 2004). Furthermore, unlike laser light, it does not penetrate the object surface, thus making it better suited to the imaging of textures such as marble (Godin et al. 2001. On the other hand, being based on projected light, the structured light systems are not effective in daylight and must be performed in a darkened environment.

\subsubsection{Depth Scanning}

While laser scanning, whether by point or by line, and white light "structured light" scanning comprise the main streams of non-invasive, non-contact $3 d$ scanning technologies around today, the search is on for new innovative approaches, which will bring $3 d$ scanning to the masses both in price and simplicity. No doubt the years to come will bring many such developments, however one direction which is already showing promise is the harnessing of consumer game console based technologies, chief amongst these the Microsoft Kinect.

As we have already learned, the key to $3 d$ scanning is a visual capture system which includes depth perception, together with software which can interpret this information and translate it into a point cloud or polygon mesh.

The Kinect sensor, now in its second generation, is in fact a game-oriented RGB-D camera, the visual receptor of the Microsoft X-Box. Whilst the $2^{\text {nd }}$ generation Kinects is based on Time of Flight technology; the original Kinect was the first general consumer-grade structured-light camera. (Orrego 2012) composed of RGB and IR sensors with an IR pattern projector (Comb`es et al. 2011).

To gauge the depth, the system uses stereo triangulation. However, whereas stereo triangulation usually requires two images, the uniqueness of this technique lies in the fact that there is only one projection system, the infrared sensor. The second pattern is hardcoded into the chip logic. The images which result are not identical as there is some distance between the two. The software recognizes the images as originating from different camera positions, thus allowing stereo triangulation to calculate the $Z$ axis position for each point.

\subsubsection{Limitations of 3D Scanners}

Clearly, any system based on viewing reflected light will suffer the same limitation as photography, 
namely, that it can record only that which it can see. Therefore, 3D scanners must overcome this limitation by either revolving the object and scanning from multiple directions, or by rotating around the object.

A second, and more serious limitation of laser scanning lies in the type of material it can record. Due to the technique being based on reflected light, laser scanners are notoriously problematic at scanning shiny surfaces, especially glass, silver, gold, marble and other reflective materials.

This limitation is often partially overcome by spraying the subject with an anti-reflective powder, much to the chagrin of museum curators and conservation specialists.

\section{TEST CASES}

To account for a representative cross-cut of real-life needs, a wide variety of objects were chosen. Differing in both size, colour, material, texture, complexity and access, these reflected the varied daily activities of documentation personnel, curators, conservators and research specialists. Where possible, each object was imaged by several methods. Where this was not possible, due to practical limitations, only one or two methods were tested.

The results of each imaging technique on each sample object were gauged and evaluated by the local specialists.

- $18^{\text {th }}$ century Ethiopian Medical Book. An illuminated manuscript with a unique open binding. Wood, parchment and string. Israel Museum, Jerusalem. $13 \times 16 \times 10 \mathrm{~cm}$. Studio.3D web link: https://skfb.ly/YAot

- $19^{\text {th }}$ century Tombstone. Jewish cemetery of Unsleben, Germany. Approx. 40×50×11 cm. Imaged in situ outdoors. 3D web link: https://skfb.ly/ODYY

- $20^{\text {th }}$ century Venetian Mask. Papier-mâché. Private collection. $42 \times 30 \times 13 \mathrm{~cm}$. Studio. 3D web link: https://skfb.ly/FVU7

- Terracotta figure. Arts of Africa, Oceania, and the Americas. Israel Museum, Jerusalem. Approx. $60 \times 40 \times 40 \mathrm{~cm}$. Imaged in situ in museum gallery. 3D web link: https://skfb.ly/DnFB

- Manuscript "Yusuf and Zulaikha", by Hakim Nuruddin Jami (1414-92). $16^{\text {th }}$ century. Far Eastern (Persian) Paper with gold leaf, Lapis Lazuli and Verde Greens. Israel Museum, Jerusalem. $26.5 \times 16.7 \mathrm{~cm}$. Studio. 3D web link: https://skfb.ly/66v8z
- Olmek Figurine. 900-600 BCE Jade. Israel Museum, Jerusalem. $18 \times 7 \times 5 \mathrm{~cm}$. Studio. 3D web link: https://skfb.ly/66uUo

- Bulla (seal) impression from Lachish treasure. Clay. $6^{\text {th }}-7^{\text {th }}$ century BCE, Israel Antiques Authority. $13 \times 15 \mathrm{~mm}$. Studio.

- Hellenistic Administration Stele. $2^{\text {nd }}-1^{\text {st }}$ century BCE. Stone. Israel Museum Jerusalem. $80 \times 60$ $\mathrm{cm}$. Imaged in situ in museum gallery. 3D web link: https://skfb.ly/66vHF

- Pipe Smoker. Bronze. $20^{\text {th }}$ century. Israel Museum, Jerusalem. $90 \times 60 \times 60 \mathrm{~cm}$. Imaged in situ outdoors. 3D web link: https://skfb.ly/YODK

- Babylonian Tablets. $6^{\text {th }}-5^{\text {th }}$ century BCE, Clay. Bible Lands Museum, Jerusalem. Approximately $5.5 \times 3.5 \times 1.5 \mathrm{~cm}$. Studio shots. 3D web links: https://skfb.ly/FnID \& https://skfb.ly/yAWN

- Roman Coin. Metal. Private collection. 1.5×1.1 $\mathrm{cm}$. Studio. 3D web link: https://skfb.ly/6nVAR

- Prayer book: Day of Atonement. London, 1806. Leather. $26 \times 18 \times 4.2 \mathrm{~cm}$. Private collection. Studio. 3D web link: https://skfb.ly/YH8X

\section{OVERVIEW OF RESULTS}

Following is an overview of results and conclusions. Limitations of space in this paper prevent providing a detailed analysis of each object. Therefore, we have attempted to give an overall evaluation of each technology in respect to the various contexts of size, material, imaging environments, regarding geometry and texture accuracy, with specific references to the test objects where possible.

\subsection{Reflectance Transformation Imaging}
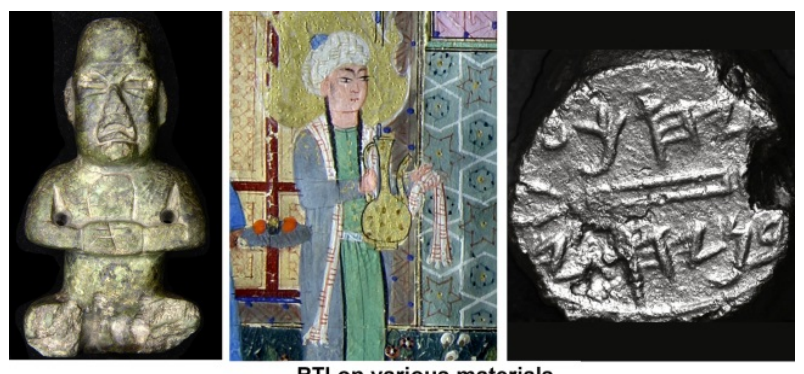

Figure 1: RTI on Olmek figurine, detail of "Yusuf \& Zulaikha", and Lachish Bulla

When it comes to harvesting fine texture information, nothing can beat the price performance of RTI. It works equally well on small or large objects, on parchment or jade (Figure 1). Like any technique, absolute attention must be placed on accuracy in shooting perfectly aligned shots, positioning the reflective ball and so on. However, with the camera sensor and lens quality available today, incredible surface details may be achieved and information invisible to the human eye may be clearly brought out, such as the virtually indecipherable fine chiseling on 
the Olmek figurine, which determine its authenticity and set it apart from forgeries, in which the details were created by metal tools (Fleitman), or the revealing of faded texts on the Hellenistic Stele (Heinrichs) (Figure 2). In the case of the Ethiopian medical book, RTI revealed previously undetected marks of an earlier binding, raising questions about its lineage (Figure 2).

Whilst strong daylight can interfere, camera aperture and shutter controls can overcome this limitation too (Figure 7). RTI is cheap and flexible. Admittedly, building a sophisticated computer controlled LED lighting dome is beyond the scope of many institutions, but cheap alternatives can work equally well, even if less efficiently.

On the downside, as each RTI demands 48-70 images and processing, if we wish to examine the surface texture details from multiple directions it can prove impractical. This is where a true 3D object proves superior.

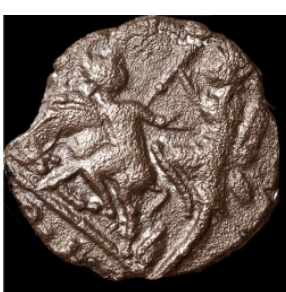

RTI details: Roman coin

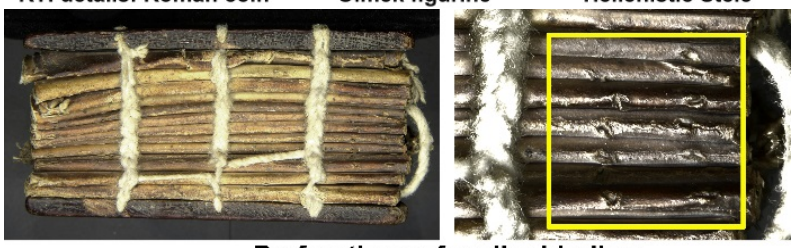

Perforations of earlier binding

Figure 2: RTI details.

\subsection{D Scanners}

Active $3 d$ laser scanners such as the NextEngine excel in surface geometry and give good detail (Figure 3) within the above-mentioned limitations. However, their effectiveness is limited to small medium sized objects and the shooting environment must be highly controlled, i.e. no daylight. One practical advantage lies in the ability to create custom-made accurate packaging for transportation.

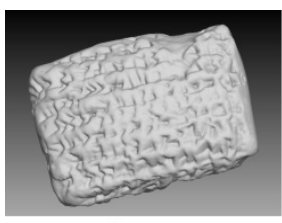

Laser

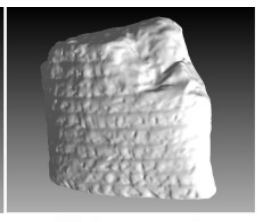

Photogrammetry

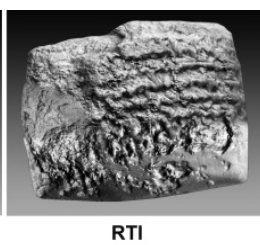

RTI
Figure 3: Sumerian tablets. Mesh geometry. Laser, Photogrammetry and RTI.

In terms of our defined cost parameters they are not cheap and lie at the very top scale, possibly outside the budget of many small institutes. Laser scanners often offer very poor or sometimes even no RGB texture. Therefore, it is important to define what our needs are and whether our priorities lie in accuracy of geometry, necessary for conservation and research, or the overall aesthetic photographic appearance necessary for public display (Figure 4).

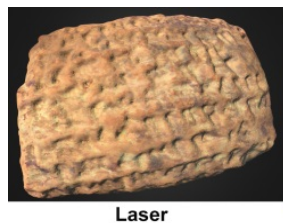

Laser

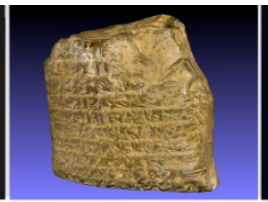

Photogrammetry

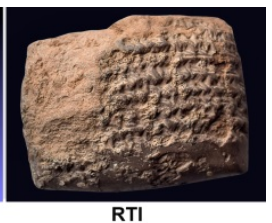

RTI
Figure 4: Sumerian tablets. Texture quality. Laser, Photogrammetry and RTI.

In our tests, too, scanner results were greatly dependent on the material qualities. Shiny, translucent and reflective surfaces such as marble, gold leaf or jade proved virtually impossible to capture by active scanning because the laser is reflected in multiple directions (Venetian mask). Likewise, dark objects (prayer book, coin) absorbed the light and very little was reflected. The usual solutions such as powders or sprays are not practical as they both change the apparent surface properties of the object and in addition can cause highly unwanted responses from the heritage community (Figure 5).

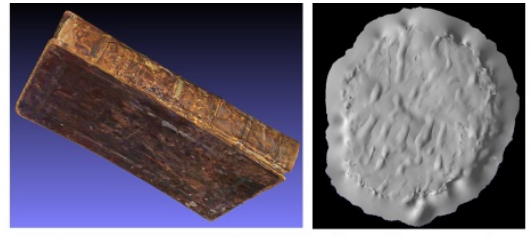

Laser scans: Prayer book, Roman coin,

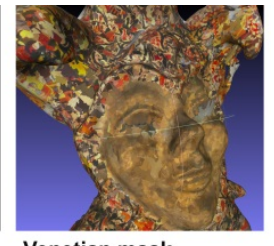

Venetian mask
Figure 5: Laser scans. Difficult surfaces.

It should be noted that this field is developing rapidly and as of the writing of this paper there are literally tens of scanners available within this price range or below. Some use laser technology, others structured light, such as the excellent David scanner (Now the HP 3D Scan). The Kinect and Sense scanners can indeed work in subdued soft light but in our tests their overall quality was constantly inferior and frankly impractical for anything other than quick and basic curatorial 3D documentation (Figure 6).
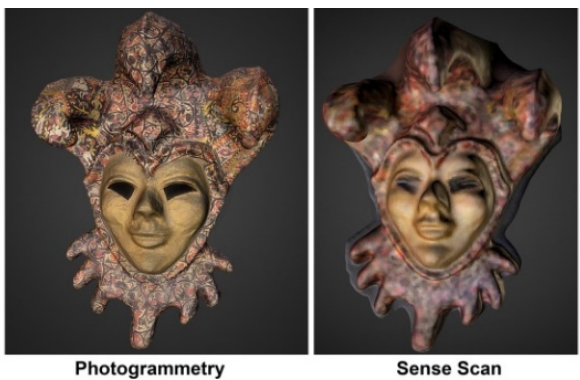

Figure 6: Venetian Mask. Photogrammetry vs Sense. 
Today, with the attention focused on depth- sensing technologies by the likes of Apple and Microsoft, we may soon expect great improvements.

\subsection{Structure from Motion - Photogrammetry}

In our tests, photogrammetry proved itself to be a general all-rounder in terms of performance. With today's cameras and software such as Agisoft Photoscan, 3D SOM or Autodesk Remake, one can achieve remarkably highly detailed point-clouds, meshes and solid surfaces. Photogrammetry works equally well on virtually any size, be it a large archaeological site recorded by drone photography, a medium sized statue or a macro object such as a coin or bulla. In the case of the Lachish Bulla, "RTI enabled considerable improvements in deciphering the faded texts, well beyond that which was achievable during the earlier research in the 1960's" (Mendel) (Figure 1).

The shooting environment too is flexible. Thus, outdoors (tombstone, pipe smoker), gallery or studio (book), all proved equally receptive (Figure 7).

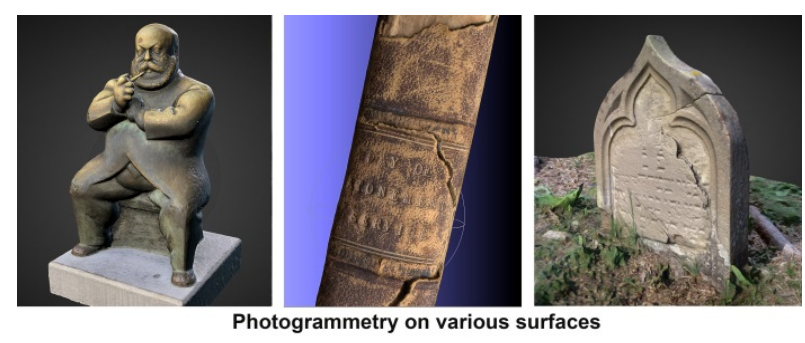

Figure 7: Photogrammetry on Bronze, Leather and Stone. Outdoors and indoors.

The basic rule is "let there be light", preferably soft light. Visual appreciation of texture information and $3 \mathrm{D}$ surface modelling is dependent on controlled directional light. Therefore, it is highly advisable to strive for a shadow less shooting environment. This however is not always possible. Where there is strong directional light (terracotta figure) it is baked onto the model, whereas if the lighting is soft and equal it may be controlled interactively post factum, (Ethiopian Medical book) rather akin to RTI, through software (Figure 8).

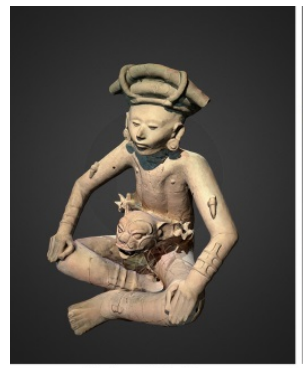

Baked light

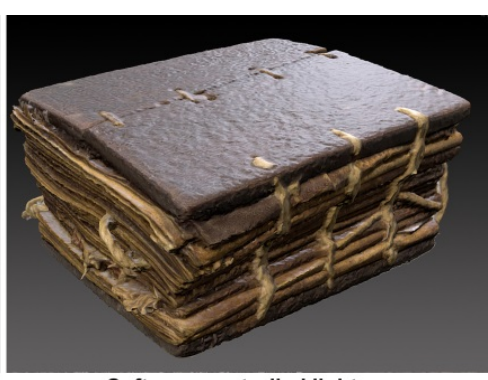

Software controlled light
Figure 8: Baked light vs software controlled light
In this respect, the 3D model has a clear advantage over RTI in that not only can the light direction be controlled but also the position and orientation of the model (pipe smoker). Where this technique stands out is of course the photographic texture or UV map. Nothing comes close to the near perfect photo rendering that can be achieved if care is taken with proper lighting, camera control and shooting technique (Figure 9). Like all things, nothing is as simple as it may seem at first. Practice and attention to detail are as always the essential imperatives to yielding a good result.

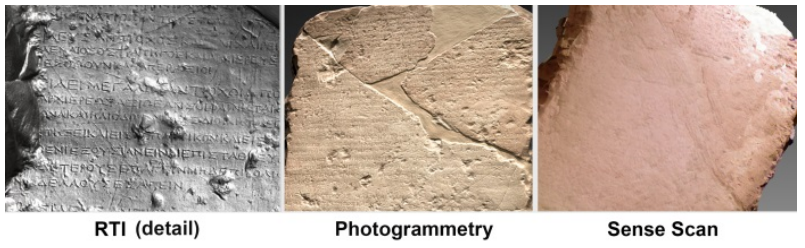

Figure 9: Hellenistic Stele. RTI, Photogrammetry, Sense.

\section{CONCLUSIONS}

Comparing the relative merits and weaknesses of the three technologies above is akin to comparing apples and oranges. Each have advantages over the other as well as clear disadvantages.

It is the opinion of this paper that all heritage institutions should make the effort to familiarize themselves with all three technologies and not form prior conclusions as to the preference of one over the other.

Furthermore, whist all the techniques are affordable, it must be stressed that attention to detail and quality in all stages of production are essential. Mastering the techniques demands time and endless testing. In RTI and SfM, the digital images should be initially captured at the highest possible resolution and in RAW format. Always remember that the final quality will be determined by the weakest link.

As for 3D scanning, price performance currently undermines its effectiveness. Lasers offer good mesh quality but poor texture. Good structured light offers better texture but at a price. the stage is set for major developments soon. Companies such as Dell, Microsoft and Apple are introducing 3D sensing technology into their computers and smart phones. Demand pushes the quality up and the prices down. So, whereas currently, laser and depth-sensing scanners are less cost effective than the alternatives, it is felt that this situation may change dramatically. 


\section{REFERENCES}

Agisoft Photoscan. http://www.agisoft.com (retrieved 4 February 2017).

Autodesk ReCap 360.

www.autodesk.com/products/recap/overview (retrieved 4 February 2017).

Autodesk Remake. https://remake.autodesk.com (retrieved 4 February 2017).

Akca, D. (2012) 3d Modeling of Cultural Heritage Objects with a Structured Light System. Mediterranean archaeology and Archaeometry, vol. 12, no 1, pp.139-152.

Bayram, B., Nemli, G., Özkanc, T., Oflazb, O.E., Kankotan, B., and Çetin, I. (2015) Comparison of Laser Scanning and Photogrammetry and their use for Digital recording of Cultural Monument Case study: Byzantine land walls-Istanbul. ISPRS Annals of the Photogrammetry, Remote Sensing and Spatial Information Sciences, Volume ii-5/w3, 25th International Cipa. Taipei, Taiwan.

Boehler, W. and Marbs, A. (2004) 3d Scanning and Photogrammetry for Heritage Recording: A Comparison. Proc. 12th Int. Conf. on Geoinformatics - Geospatial Information Research: Bridging the Pacific and Atlantic, University of Gävle, Sweden.

Bourke, P. (2016) Report: Comparing Laser Scanning to 3D Reconstruction.

http://paulbourke.net/miscellaneous/laservs3d/

Cultural Heritage Imaging.

http://culturalheritageimaging.org/Technologies/RTI (retrieved 14 February 2017).

Grussenmeyer, P., Landes, T., Voegtle, T., and Ringle, K. (2008) Comparison Methods of Terrestrial Laser Scanning, Photogrammetry and Tacheometry Data for Recording of Cultural Heritage Buildings. The International Archives of the Photogrammetry, Remote Sensing and Spatial Information Sciences. vol. xxxvii. part b5. Beijing, China.

Hassani, F. (2015) Documentation of cultural Heritage Techniques, Potentials and Constraints. the international archives of the photogrammetry, remote sensing and spatial information sciences, volume $\quad \mathrm{xl}-5 / \mathrm{w} 7$. 25th International Cipa Symposium. Taipei, Taiwan.

HP. 3D Scan.

http://www8.hp.com/us/en/campaign/3Dscanner/ov erview.html (retrieved 2 March 2017).

Kadobayashi, R, Kochi, N., Otani, B., and Furukawa, R. (2004) Comparison and Evaluation of Laser Scanning and Photogrammetry and their Combined Use for Digital Recording of Cultural Heritage. national institute of information and communications technology, Seika-cho, Sorakugun, Kyoto 619-0289, Japan.
Macdonald, L. W. (2011) Visualising an Egyptian Artefact in 3D: Comparing RTI with Laser Scanning. In J. P. Bowen, S. Dunn, \& K. Ng (eds.), EVA London 2011: Electronic Visualisation and the Arts. BCS, Electronic Workshops in Computing, pp. 155-162.

Malzbender, T., Gelb, D., and Wolters, H. (2001) Polynomial Texture Maps, Proc. ACM SIGGRAPH, 28, pp. 519-528.

Meshlab. http://www.meshlab.net (retrieved 4 February 2017).

Pavelka, K., Řezníček, J., Bílá, Z., and Prunarová, L. (2013) Non-Expensive 3d Documentation and Modelling of Historical Object and Archaeological Artefacts by Using Close Range Photogrammetry. Geoinformatics FCE CTU.

Payne, E. M., (2013). Imaging Techniques in Conservation. Journal of Conservation and Museum Studies, 10(2), pp.17-29. DOI: 10.5334/icms. 1021201

Reznicek, J., Pavelka, K. (2008) New Low-Cost 3d Scanning Techniques for Cultural Heritage Documentation. The International Archives of the Photogrammetry, Remote Sensing and Spatial Information Sciences. Vol. xxxvii. Part b5. Beijing, China.

RTI viewer.

http://culturalheritageimaging.org/What We Offer/ Downloads/index.html (retrieved 8 January 2017).

Scopigno, R., Callieri, M., Cignoni, P., Corsini, M., Dellepiane, M., Ponchio, F., and Ranzuglia, G. (2011) 3d Models for Cultural Heritage: Beyond Plain Visualization. IEEE Computer Society.

Skarlatos, D. and Kiparissi, S. (2012) Comparison of Laser Scanning, Photogrammetry and SfM-MVS Pipeline Applied in Structures and Artificial Surfaces. Annals of the Photogrammetry, Remote Sensing and Spatial Information Sciences, Volume i-3.

Wachowiak, M. J. and Karas, B. V. (2009) 3d Scanning and Replication for Museum and Cultural Heritage Applications, Jaic 48.

\section{ACKNOWLEDGEMENTS}

Chelsea Graham. Institute for the Preservation of Cultural Heritage. Yale University. RTI image of tombstone, Germany.

Yvonne Fleitman. Benjamin Weiss Curator, Art of the Americas, Israel Museum, Jerusalem.

Johannes Heinrichs. Koeln University, Germany.

Anat Mendel-Geberovich. Institute of Archaeology and School of Physics and Astronomy. Tel Aviv University. 